\title{
Analysis of a Device for Texturing by Burnishing Using Principles from Axiomatic Design
}

\author{
Gheorghe Nagîț, Laurenţiu Slătineanu, Vasile Merticaru, Marius Ionuţ Rîpanu, Andrei Marius Mihalache, \\ Lucian Tăbăcaru, and Mihai Boca \\ “Gheorghe Asachi” Technical University of Iași, Bvd. D. Mangeron 39A, Iași, 700050, Romania
}

\begin{abstract}
The vibro-burnishing is a process by means a ball or a roller or other tool active surface is pressed and moved, inclusively by applying a vibratory motion, along the surface to be finished, generating a specific microrelief. A device for vibro-burnishing adapted on a universal lathe was analyzed by means of some principles corresponding to the Axiomatic Design theory. The analysis was developed by adopting some simplifying assumptions and it proved an adequate design, by considering some functional requirements and design parameters specific to the device.
\end{abstract}

\section{Introduction}

Frequently, within mechanical equipment, the surfaces by which a part contacts another part must have a low roughness; for example, if there is an interference fit, the recommended values for the surface roughness parameter $R a$ are of 3.2-6.4 $\mu \mathrm{m}$, while for the clearance fit, the surface roughness parameter $R a$ has values lower than $1.6 \mu \mathrm{m}$. It is interesting the fact that sometimes, in the case of clearance fits, there is the recommendation that the surface has a certain microrelief, necessary in order to fulfil the condition of retaining the oil applied for diminishing the friction forces. Thus, one can meet situations when after a final machining process of grinding or another finishing process, a certain supplementary machining process must be applied, in order to generate the small cavities able to retain the oil.

There are distinct machining processes which can be used in order to generate the desired microrelief on the part surfaces for the above mentioned situations; if the main characteristics of the processes are taken into consideration, one can classify these machining processes as being based on cutting (abrasive) phenomena and on surface plastic cold deformation, respectively. In the group of machining methods based on cold plastic superficial deformation, there is a process called texturing by burnishing. Essentially, such a process is materialized by pressing and moving the active part of a plastic deformation tool along the preestablished trajectories on the surface of the metallic part to be machined. If a vibration is used in order to obtain the desired superficial texture and a roll or a tool similar to a roll is used, the process could be named "vibro burnishing" or "vibro-rolling".

Over the years, the texturing by burnishing constituted an objective for the researches in the field of machine manufacturing technology.
Thus, some decades ago, an ample research of this machining method was developed and promoted in the former Soviet Union by G. Schneider [1]; he proposed a set of devices for investigating the influence of distinct factors on the properties of the surface layer obtained by burnishing and vibro-burnishing processes.

Revenko et al. [2] were interested to investigate the influence of the microrelief obtained by ball vibroburnishing of a steel part upon the corrosion resistance. They noticed that in comparison with the grinding, the vibro-burnishing ensures a better part behavior to the corrosion process in a humidity cabinet and in a saltspray camera.

Kumar and Purohit took into consideration the possibilities of developing an improved ball burnishing tool, which could be positioned and clamped in the tool holder of a universal lathe [3]. They considered that this tool is convenient for developing a research concerning the influence exerted by some process input factors (workpiece rotation speed, feed, force, number of passes) upon the values of the parameters of technological interest.

Hadamache et al. assumed the objective to develop a device for mechanical plastic deformation by means of a ball or a roller burnishing [4]. They concluded that if the surface roughness, the hardness and the wear resistance are taken into consideration as process outputs, the roller burnishing offers a better surface roughness, while the ball burnishing is advantageous when the increase of the surface layer hardness is considered as final objective.

Korzynski and Lubas approached the problem of obtaining a certain bushing texturing by burnishing [5] They developed a theoretical and experimental research using an oscillatory movement along the longitudinal feed in the case of finishing internal cylindrical surfaces. Distinct constructive solutions were designed in order to

\footnotetext{
Corresponding author: nagit@tcm.tuiasi.ro
} 
obtain a more adequate fulfilling of the machining process requirements.

Within the "Gheorghe Asachi" Technical University of Iași, a research developed by Nagit highlighted the possibility of using a ball vibro-burnishing process and a diamond type tool also within a vibro-burnishing process, in order to diminish the surface roughness and to improve the service properties of steel parts [6].

On the other hand, the Axiomatic Design theory takes into consideration the so-called functional requirements in order to adequately establish the design parameters and process variables; this design theory was used in the last years in order to design various mechanical equipment or to analyze distinct versions of solving various problems [7-10].

The objective followed by this paper was to present the results obtained by using the principles of the Axiomatic Design for analyzing a device for vibroburnishing adapted on a universal lathe.

\section{Theoretical aspects concerning the process of vibro-burnishing}

As a consequence of applying distinct machining processes involving the material removal from the workpiece, asperities with various heights are generated on the machined surface. Usually, such machining methods determines also the change of some properties of the surface layer; the crystals are deformed and sometimes there is also a heat affected zone, as a result of high temperature developed, for example, when high machining speeds are used. In order to diminish the height of asperities obtained by previous applied machining process and, simultaneously, to increase the hardness of the superficial metallic layer, a burnishing process could be applied. In such a case, a ball is placed instead the cutting tool specific to a longitudinal turning process. This ball is supported by one or more rolling elements and it is pressed on the surface to be burnished. In order to increase the process efficiency, a vibratory additional movement could be achieved by the ball and, in this way, a vibro-burnishing process could be materialized (Figure 1).

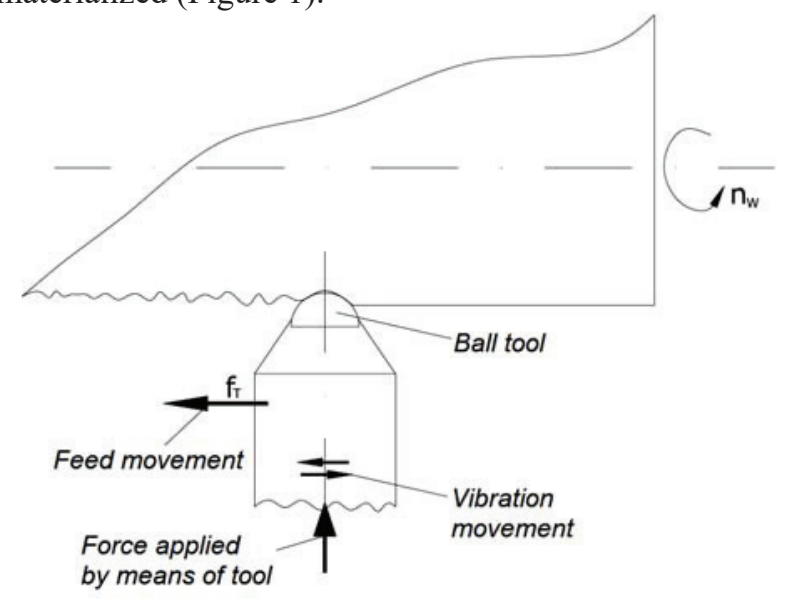

Fig. 1. Machining scheme valid in the case of vibro-burnishing on a lathe.
In the work zone, the ball presses the workpiece material found in asperities, achieving a smoothing effect; practically, as a consequence of the deformation process, the material of asperities is re-distributed, so that a decrease of the asperities height is achievable (Figure 2). A second effect of the process is the strong deformation of the crystals found in the surface layer; evidently, the crystals found near the workpiece surface are affected by a more intense plastic deformation process, while at a higher distance from the workpiece surface, crystals are practically not affected by the vibroburnishing process.

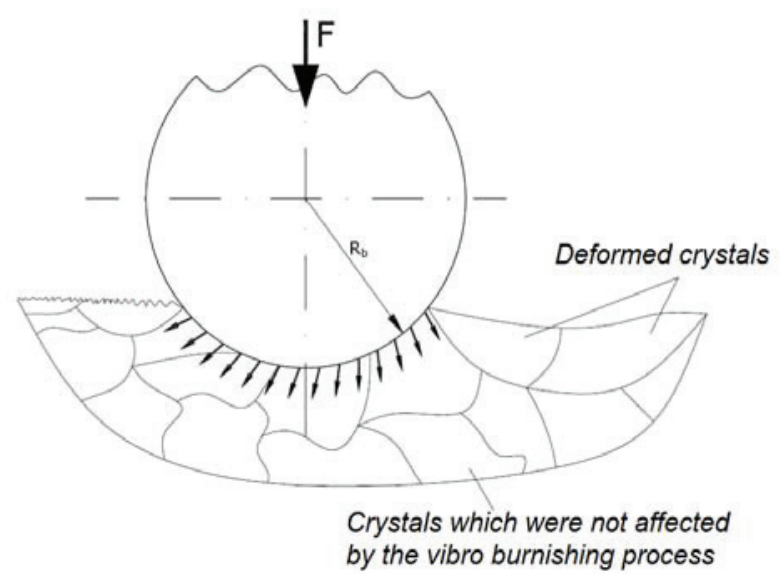

Fig. 2. Phenomena developed in the work zone in the case of vibro-burnishing.

In order to materialize the vibro-burnishing process and, in accordance with the above mentioned aspects, a device for vibro-burnishing adaptable on a universal lathe was designed and achieved [6]. The nominated device (Figure 3 ) includes a plate which can be placed instead of the common tool holder existing on the universal lathes. An electric motor generates a rotation movement which is transmitted by means of transmission belts to a shaft able to rotate a disk. On this disk, a cylindrical bolt could be moved along a radial direction by acting a drive screw; in this way, the bolt could be placed at distinct radial distances. The eccentric bolt is linked to a connecting rod, which determines a bar to achieve a rectilinear movement, together with the support of the burnishing tool. The bar is supported by another rod, pressed by compressing a spring able to generate a force whose value could be read due to a piece which indicates the deformation of the spring.

The tool is constituted in this case by a ball supported by a radial bearing ball, able to ensure the easy rotation of the ball found in contact with the workpiece surface.

The main work parameters are: the speed of workpiece rotation $n_{W}$ [rev./min.], the speed of longitudinal feed movement $f_{T} \quad[\mathrm{~mm} / \mathrm{min}$.$] , the$ force/pressure $F / p$ exerted by the ball on the workpiece surface, the amplitude $A$ and the frequency $f$ of vibratory movement achieved by the ball. In order to develop a research concerning the influence of the process input factors on the process results, it was necessary to ensure possibilities of modifying the values of the input factors between certain limits. 


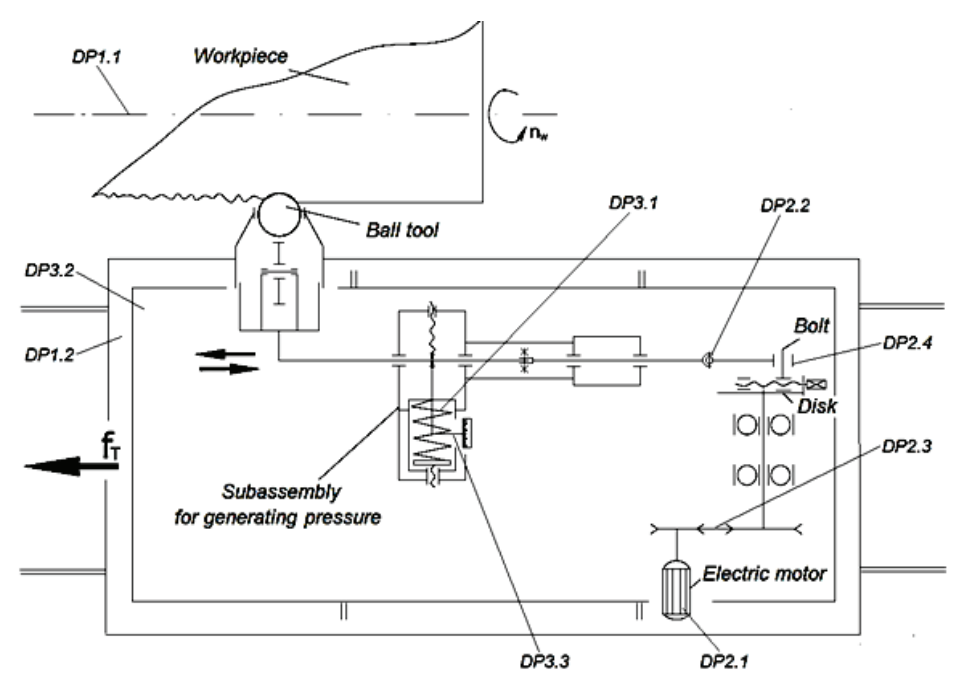

Fig. 3. Kinematic scheme of the device for vibro-burnishing on a lathe.

Thus, the rotation speed $n_{W}$ of the workpiece could be achieved by means of gear box of the universal lathe. The machine tool (universal lathe) facilitates also the easy change of the speed corresponding to the longitudinal feed speed $f_{T}$.

The pressure exerted by the ball on the workpiece surface could be changed by means of the spring included in the device. A certain compression value for the spring, obtained through a screw mechanism, determines a certain small deflection and the corresponding elastic force in the ball tool's supporting shaft, which determines the force/pressure $F / p$ exerted by the ball on the workpiece.

In order to change the frequency $f$ of the vibratory movement achieved by the tool (ball), pulleys with distinct diameters could be used. By modifying the ratio in the belt transmission, the frequency of the vibratory movement driven by the crank mechanism is changed. The amplitude $A$ of the vibratory movement could be established by positioning the bolt at a certain radial distance on the disk rotated by the electric motor. In this way, the length of the crank is modified.

\section{Principles specific to the applying of axiomatic design method}

There are distinct design methods able to facilitate the selecting by the designer the more adequate constructive solution among many various available solutions.

One of these design theories was proposed some decades ago by Nam Suh, who was then professor in the Massachusetts Institute of Technology (U.S.A.) [7]. Professor Suh tried to establish a more systematic and organized developing of the design activity; over the years, the method of axiomatic design became more and more known and applied, not strictly to solve design problems, but finding also applications in other various field of human activities.

The method takes into consideration two essential axioms:
1. Axiom of independence, which requests the independence of functional requirements corresponding to the equipment to be designed;

2. Axiom of information, which mentions that the quantity of information necessary in the design activity is minimum.

There are also four domains:

a) Customer domain, where the customer's requests are highlighted;

b) Functional domain, where the functional requirements are defined;

c) Physical domain, where the design parameters are established;

d) Process domain, where the process variables are determined.

\section{Functional requirements and design parameters in the case of device for vibro-burnishing}

As above mentioned, a device for vibro-burnishing was designed and built within a doctoral research developed by Nagit [6]. Taking into consideration the appearance of the Axiomatic Design method and in order to find if some improvements could be applied to this device, an analysis of the device by means of some principles valid in the case of Axiomatic Design was developed. One must mention that in order to ensure a reasonable length of explanations and an easier understanding the way of using the principles of Axiomatic Design, some simplifying assumptions concerning the constructive solution were applied.

Thus, one could consider that the customer (researcher) needs a device for developing a research activity concerning the vibro-burnishing of external cylindrical surfaces belonging to metallic workpieces.

As customer needs, one can mention:

CN1: the device could be achieved within a laboratory for mechanical machining, being adaptable on one of the available machine tools; 
CN2: in order to ensure conditions for an experimental research, some possibilities to change the values of the vibro-burnishing process input factors must be ensured.

In accordance with the above mentioned customer needs, the main functional request is:

FRO: design a device for vibro-burnishing, adaptable on one of the machine tools available in a mechanical workshop/laboratory.

If the functional requirements (FRs) of first order must be formulated, these can be:

FR1: use a machine tool available in the laboratory, able to ensure a rotation movement and a longitudinal feed movement;

FR2: ensure a vibratory movement, with preestablished values of frequency $f$ and amplitude $A$;

FR3: ensure pre-established values for the pressure $p$ exerted by ball tool on the workpiece cylindrical surface;

When the problem of defining the functional requirements of second order is formulated, one can arrive to:

FR1.1: use the rotation movement of the machine tool main shaft;

FR1.2: use the feed movement of the machine tool table/subassembly;

FR2.1: use a supplier of rotation movement, usually easier obtained by means of electric motors;

FR2.2: transform the rotation movement in a rectilinear one;

FR2.3: ensure possibilities of changing the rotation speed;

FR2.4: ensure possibilities of changing the amplitude $A$ of the vibratory movement;

FR3.1: include element for generating the force $F$;
FR3.2: ensure possibilities for changing the value of the force $F$;

FR3.3: ensure possibilities for highlighting the value of the force/pressure $F / p$ exerted by the ball on the workpiece.

In accordance with the above mentioned functional requirements, the following design parameters $(D P S)$ were identified:

DP1.1: main shaft of the universal lathe;

DP1.2: lathe apron;

$D P 2.1$ : electric motor;

DP2.2: crank mechanism;

$D P 2.3$ : belts transmission;

DP2.4: radial slide for moving the bolt corresponding to the connection between the crank and connecting rod;

DP3.1: spring;

DP3.2: transversal slide of the machine tool;

DP3.3: bolt which highlights the value of the spring compression, on a graduate ruler.

These functional requirements and design parameters were included in the Table 1, in order to check the validity of the independence axiom.

The correspondence between the functional requirements FRs and design parameters DPs could be written as a matrix relation:

$$
\{F R\}=[A]\{D P\},
$$

where $A$ is the design matrix by which the transfer functions $F R$ and $D P$ are taken into consideration.

In the case of the identified device for vibroburnishing, the relation (1) could be written by using Table 1, as in equation (2).

Table 1. Functional requirements and design parameters.

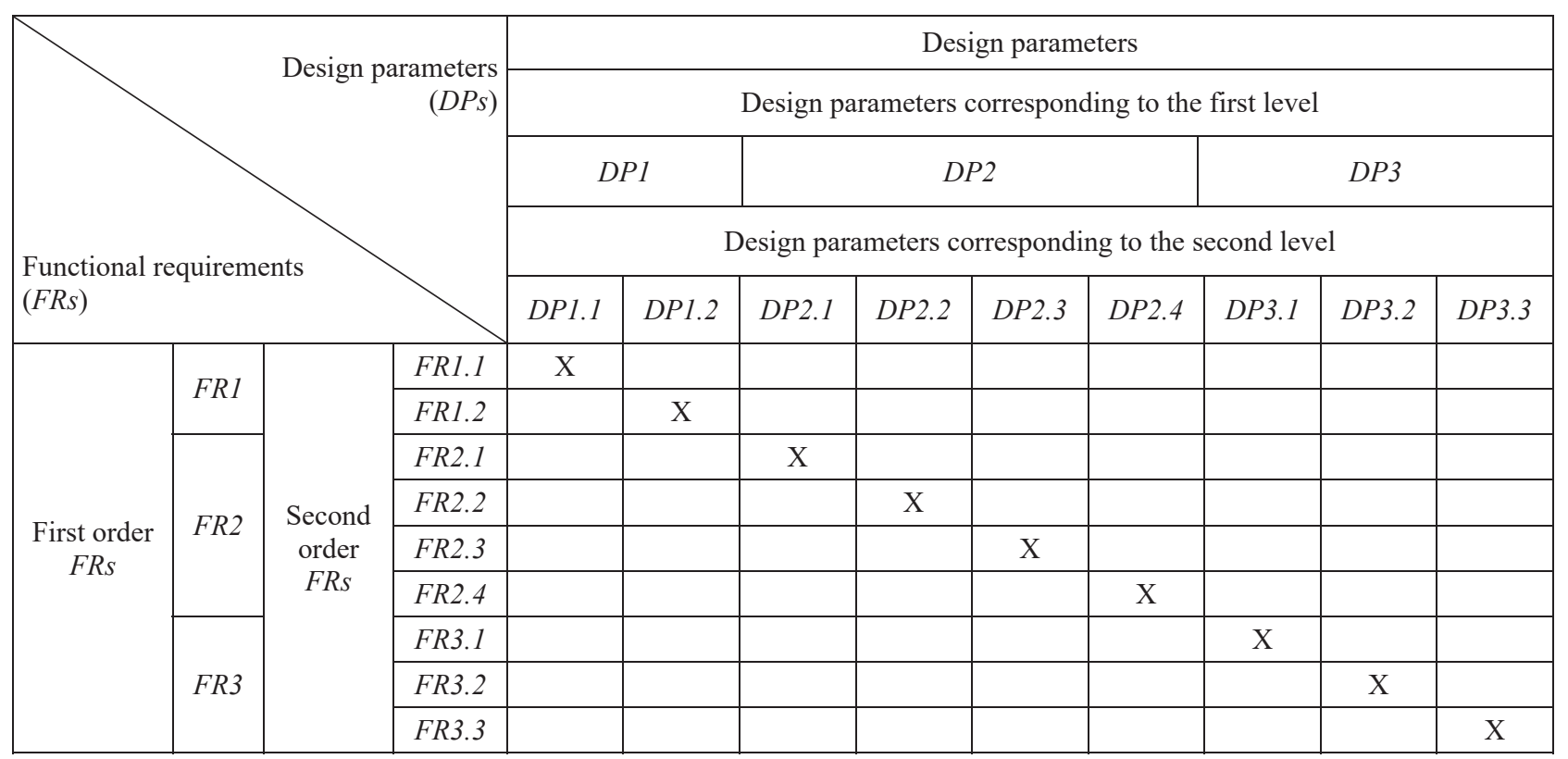




$$
\left\{\begin{array}{l}
F R 1.1 \\
F R 1.2 \\
F R 2.1 \\
F R 2.2 \\
F R 2.3 \\
F R 2.4 \\
F R 3.1 \\
F R 3.2 \\
F R 3.3
\end{array}\right\}=\left[\begin{array}{ccccccccc}
X & 0 & 0 & 0 & 0 & 0 & 0 & 0 & 0 \\
0 & X & 0 & 0 & 0 & 0 & 0 & 0 & 0 \\
0 & 0 & X & 0 & 0 & 0 & 0 & 0 & 0 \\
0 & 0 & 0 & X & 0 & 0 & 0 & 0 & 0 \\
0 & 0 & 0 & 0 & X & 0 & 0 & 0 & 0 \\
0 & 0 & 0 & 0 & 0 & X & 0 & 0 & 0 \\
0 & 0 & 0 & 0 & 0 & 0 & X & 0 & 0 \\
0 & 0 & 0 & 0 & 0 & 0 & 0 & X & 0 \\
0 & 0 & 0 & 0 & 0 & 0 & 0 & 0 & X
\end{array}\right]\left\{\begin{array}{l}
D P 1.1 \\
D P 1.2 \\
D P 2.1 \\
D P 2.2 \\
D P 2.3 \\
D P 2.4 \\
D P 3.1 \\
D P 3.2 \\
D P 3.3
\end{array}\right\}
$$

In accordance with the theory of Axiomatic Design, the matrix corresponding to the design matrix is a diagonal matrix and it is considered as uncoupled matrix; this could be appreciated as a result of a good design. One must mention that in an initial version considered for the design matrix, in the case of DP1.1, $D P 1.2$, and $D P 2.4$, the lathe was considered and such a version led to a coupled matrix and coupled design, which are not appreciated as adequate from the point of view of the axiomatic design. A second analysis proved the fact that the functional request FR1.1, FR1.2, FR2.4 are really materialized by means of distinct subassemblies of the lathe and, in this way, an uncoupled matrix could be used.

In the sense of getting forward with the use of Axiomatic Design theory, distinct versions could be searched for each design parameter $D P$ and subsequently changes could affect the functional requirements FRs.

Another possibility to develop the use of the Axiomatic Design theory could take into consideration the possibilities to split the functional requirements in order to adequately justify the distinct components of the device.

\section{Conclusions}

The vibro-burnishing is a machining method by which the workpiece surface layer obtained by other previously applied machining techniques is pressed by means of a ball or of other tool active surface, so that a certain relief (usually a relief characterized by a lower surface roughness) could be obtained. The pressure applied by the tool active zone determines a process of plastic deformation, accompanied by an increase of the microhardness and this effect could determine the improvement of final part service properties.

In order to develop a research concerning the influence of distinct process input factors on the values of parameters of technological interest valid in case of vibro-burnishing, a device adaptable on a universal lathe was designed and built.

Afterwards, as a result of appearance and development of the Axiomatic Design theory, the problem of analyzing the measure in which the built device corresponds to the principles of the Axiomatic Design is formulated.

In this way, a so-called uncoupled matrix is identified for a simplified application of some considerations corresponding to the Axiomatic Design theory and this could have the significance of a good design.

In the future, the analysis could be continued by taking into consideration other components and subassembly of the device for vibro-burnishing, in order to identify eventual possibilities of improving its constructive solution and, subsequently, the process variables could be established.

\section{References}

1. G. Schneider, Properties of machine parts with regular micro-reliefs (in Russian), (Maschinostroenie, Leningrad, 1982)

2. V.G. Revenko, G.R. Alekseeva, N.F. Stepanov, N.L. Bogdashkina, G.P. Chernova, Protection of Metals, 38, 1 (2002)

3. P. Kumar and G.K. Purohit, International Journal of Engineering Research and Technology, 6, 6 (2013)

4. H. Hamadache, L. Laouar, N.E. Zeghib, K. Chaoui, Journal of Materials Processing Technology, 180, (2006)

5. M. Korzynski, J. Lubas, Nonconventional finishing technologies (Polish Scientific Publishers PWN, Warsaw, 2013)

6. G. Nagît, Theoretical and experimental contributions concerning the machining by vibroburnishing (in Romanian), ("Gheorghe Asachi" Technical University of Iași, 1997)

7. N.P. Suh, Axiomatic Design: Advances and Applications (Oxford University Press, New York, 2001)

8. C.A. Brown, Journal of Manufacturing Systems, 24, 3 (2005)

9. M.K. Thompson, Introduction to Axiomatic Design Theory, Tutorials of The Eight International Conference on Axiomatic Design, ICAD 2014.

10. L. Slătineanu, Industrial property (in Romanian), (Performantica Publishing House, Iaşi, 2015) 